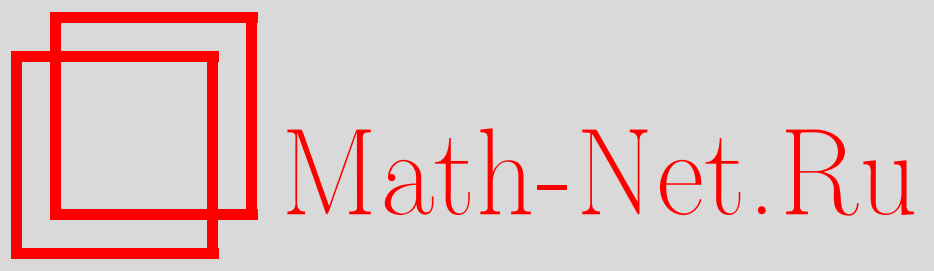

В. Г. Марихин, Квазиштеккелевы системы и двумерные уравнения Шредингера в электромагнитном поле, ТМФ, 2013, том 177, номер 1, 83-92

DOI: https://doi.org/10.4213/tmf8540

Использование Общероссийского математического портала Math-Net.Ru подразумевает, что вы прочитали и согласны с пользовательским соглашением http://www . mathnet.ru/rus/agreement

Параметры загрузки:

IP: 3.81 .55 .215

26 апреля 2023 г., 14:02:43

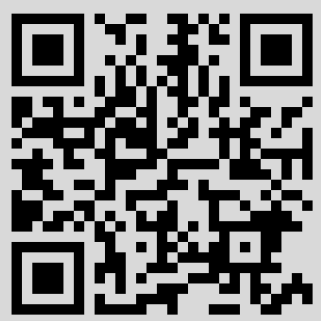




\title{
КВАЗИШТЕККЕЛЕВЫ СИСТЕМЫ И ДВУМЕРНЫЕ УРАВНЕНИЯ ШРЕДИНГЕРА В ЭЛЕКТРОМАГНИТНОМ ПОЛЕ
}

\begin{abstract}
Получена полная классификация двумерных уравнений Шредингера в электромагнитном поле с дополнительным квадратичным по импульсам интегралом. Для этого надо произвести замену переменных типа Ковалевской и привести гамильтонианы к квазиштеккелевой форме; в этой форме провести классификацию в смысле Пенлеве, а затем вернуться к оригинальным переменным.
\end{abstract}

Ключевые слова: квантовые волчки, интегрируемость, уравнение Шредингера.

DOI: $10.4213 / \operatorname{tmf} 8540$

\section{1. ПАРЫ КОММУТИРУЮЩИХ КВАДРАТИЧНЫХ ОПЕРАТОРОВ}

Проблема интегрируемых случаев двумерного уравнения Шредингера в электромагнитном поле изучается довольно давно (см., например, работу [1] и ссылки в ней), но до сих пор не исследована до конца. С появлением современных методов анализа интегрируемых систем были рассмотрены разные аспекты исследуемой эадачи, например, в работе [2] изучался класс (конечнозонных) решений уравнения Шредингера с магнитным полем (см. также работу [3]). В статье [1] для решения данной задачи применялся метод факторизации (см. также ссылки в этой работе). Уравнение Шредингера в магнитном поле с дополнительными линейным и квадратичным интегралами движения рассматривалось, например, в работе [4] (см. также цитированные там работы), где был получен ряд интересных примеров таких уравнений в различных системах координат, но использованный в [4] подход не претендует на полную классификацию таких систем.

В настоящей работе мы следуем схеме, изложенной в статье [5], основные положения которой мы повторим в настоящей статье, но, в отличие от работы [5], мы получим полную классификацию в оригинальных переменных, используя алгебраический факт факторизуемости уравнений, определяющих основные функции в квазиштеккелевой форме, т. е. в форме, в которой к штеккелеву гамильтониану

${ }^{*}$ Институт теоретической физики им. Л.Д. Ландау РАН, Черноголовка, Московская обл., Россия. E-mail: mvg@itp.ac.ru 
добавляются дополнительные члены, линейные по производным (в квантовом случае) или по импульсам в (классическом случае), подробнее см. ниже. Мы перейдем к переменным типа Ковалевской и получим квазиштеккелеву (каноническую) форму квадратичных коммутирующих операторов. Произведя полную класификацию (в смысле Пенлеве) пар коммутирующих операторов этого вида и введя ограничение для одной из функций от одной переменной (как следствие того, что один из первоначальных гамильтонианов был оператором Шредингера), мы перейдем обратно к уравнению Шредингера.

Рассмотрим сначала общую задачу о коммутирующих парах квантовых операторов в электромагнитном поле, квадратичных по операторам импульса. Наиболее общий вид таких пар следующий:

$$
\begin{aligned}
H= & a_{1}\left(q_{1}, q_{2}\right) \hat{p}_{1}^{2}+2 b_{1}\left(q_{1}, q_{2}\right) \hat{p}_{1} \hat{p}_{2}+c_{1}\left(q_{1}, q_{2}\right) \hat{p}_{2}^{2}+ \\
& +d_{1}\left(q_{1}, q_{2}\right) \hat{p}_{1}+e_{1}\left(q_{1}, q_{2}\right) \hat{p}_{2}+f_{1}\left(q_{1}, q_{2}\right), \\
K= & a_{2}\left(q_{1}, q_{2}\right) \hat{p}_{1}^{2}+2 b_{2}\left(q_{1}, q_{2}\right) \hat{p}_{1} \hat{p}_{2}+c_{2}\left(q_{1}, q_{2}\right) \hat{p}_{2}^{2}+ \\
& +d_{2}\left(q_{1}, q_{2}\right) \hat{p}_{1}+e_{2}\left(q_{1}, q_{2}\right) \hat{p}_{2}+f_{2}\left(q_{1}, q_{2}\right),
\end{aligned}
$$

где $\hat{p}_{1}=-i \hbar \partial_{q_{1}}, \hat{p}_{2}=-i \hbar \partial_{q_{2}}$. Мы удерживаем во всех формулах постоянную Планка $\hbar$, чтобы иметь возможность перейти к классическому случаю, который был рассмотрен в работе [6] (см. также цитируемые там работы) и в работе [7] в применении к движению заряженной частицы в электромагнитном поле. В некоторых случаях это также позволит нам строить решения получающихся уравнений в виде ряда по степеням $\hbar$.

Диагонализуем одновременно пару $H$ и $K$, т. е. оператор $K-s H$, где $s$ - параметр [6], [8]. Нетрудно видеть, что для этого должно быть выполнено следующее уравнение относительно $s$ :

$$
\Phi\left(q_{1}, q_{2}, s\right) \equiv\left(b_{2}-s b_{1}\right)^{2}-\left(a_{2}-s a_{1}\right)\left(c_{2}-s c_{1}\right)=0
$$

Решая это квадратное уравнение, получаем в общем случае два корня $s_{1}\left(q_{1}, q_{2}\right)$ и $s_{2}\left(q_{1}, q_{2}\right)$. Будем называть систему (1) невырожденной, если якобиан $\frac{D\left(s_{1}, s_{2}\right)}{D\left(q_{1}, q_{2}\right)} \neq 0$. Любая невырожденая система вида (1) путем замены переменных $\left(q_{1}, q_{2}\right) \rightarrow\left(s_{1}, s_{2}\right)$ и калибровочного преобразования $H \rightarrow g^{-1} H g, K \rightarrow g^{-1} K g$ может быть приведена к каноническому (квазиштеккелеву) виду (для упрощения обозначений мы делаем замену $\left.s_{1}=x, s_{2}=y\right)$ :

$$
\begin{aligned}
H= & \hat{\pi}_{1} \frac{S_{1}(x)}{x-y} \hat{\pi}_{1}-\hat{\pi}_{2} \frac{S_{2}(y)}{x-y} \hat{\pi}_{2}+\frac{U_{1}-U_{2}}{x-y}, \\
K= & \hat{\pi}_{1} \frac{y S_{1}(x)}{x-y} \hat{\pi}_{1}-\hat{\pi}_{2} \frac{x S_{2}(y)}{x-y} \hat{\pi}_{2}- \\
& -S_{1}(x) A_{1} \hat{\pi}_{1}-\hat{\pi}_{1} S_{1}(x) A_{1}-S_{2}(y) A_{2} \hat{\pi}_{2}-\hat{\pi}_{2} S_{2}(y) A_{2}- \\
& -S_{1}(x) A_{1}^{2}-S_{2}(y) A_{2}^{2}+\frac{y U_{1}-x U_{2}}{x-y},
\end{aligned}
$$


где

$$
\begin{array}{rlrl}
\pi_{1} & =-i \hbar \partial_{x}-A_{1}, & \pi_{2}=-i \hbar \partial_{y}-A_{2} \\
A_{1}=-\frac{1}{2} \frac{\sqrt{S_{2}(y)}}{\sqrt{S_{1}(x)}} \frac{Z_{y}}{x-y}, & A_{2}=\frac{1}{2} \frac{\sqrt{S_{1}(x)}}{\sqrt{S_{2}(y)}} \frac{Z_{x}}{x-y}
\end{array}
$$

Здесь $Z=Z(x, y)$ - функция двух переменных и

$$
\begin{aligned}
& U_{1}=V_{1}+\hbar^{2}\left[-\frac{1}{4} S_{1}^{\prime \prime}(x)+\frac{1}{16} \frac{\left(S_{1}^{\prime}(x)\right)^{2}}{S_{1}(x)}+\frac{1}{2} \frac{S_{1}^{\prime}(x)}{x-y}-\frac{3}{4} \frac{S_{1}(x)}{(x-y)^{2}}\right], \\
& U_{2}=V_{2}+\hbar^{2}\left[-\frac{1}{4} S_{2}^{\prime \prime}(y)+\frac{1}{16} \frac{\left(S_{2}^{\prime}(y)\right)^{2}}{S_{2}(y)}-\frac{1}{2} \frac{S_{2}^{\prime}(y)}{x-y}-\frac{3}{4} \frac{S_{2}(y)}{(x-y)^{2}}\right],
\end{aligned}
$$

где

$$
\begin{aligned}
& V_{1}=\frac{1}{2} \sqrt{S_{1}(x)} \partial_{x}\left[\sqrt{S_{1}(x)} \frac{Z_{x}^{2}}{x-y}\right]+f_{1}(x), \\
& V_{2}=-\frac{1}{2} \sqrt{S_{2}(y)} \partial_{y}\left[\sqrt{S_{2}(y)} \frac{Z_{y}^{2}}{x-y}\right]+f_{2}(y) .
\end{aligned}
$$

Введем также функцию $Q=\left(V_{1}^{q}-V_{2}^{q}\right) /(x-y)$, где

$$
\begin{aligned}
& V_{1}^{q}=V_{1}+\hbar^{2}\left[\frac{1}{8} \frac{S_{1}^{\prime}(x)}{x-y}-\frac{5}{16} \frac{S_{1}(x)}{(x-y)^{2}}\right], \\
& V_{2}^{q}=V_{2}+\hbar^{2}\left[-\frac{1}{8} \frac{S_{2}^{\prime}(y)}{x-y}-\frac{5}{16} \frac{S_{2}(y)}{(x-y)^{2}}\right],
\end{aligned}
$$

и функции

$$
\begin{aligned}
R_{1}= & \hbar^{2}\left[-\frac{1}{4} \partial_{x} \frac{\sqrt{S_{1}(x)}}{x-y} \partial_{x} \frac{\sqrt{S_{1}(x)}}{x-y} \partial_{x} Z+\frac{3}{8} \partial_{x} \frac{S_{1}(x)}{(x-y)^{3}} \partial_{x} Z+\right. \\
& \left.+\frac{3}{16} \frac{4 S_{1}(x)-(x-y) S_{1}^{\prime}(x)}{(x-y)^{4}} \partial_{x} Z\right], \\
R_{2}= & \hbar^{2}\left[-\frac{1}{4} \partial_{y} \frac{\sqrt{S_{2}(y)}}{x-y} \partial_{y} \frac{\sqrt{S_{2}(y)}}{x-y} \partial_{y} Z-\frac{3}{8} \partial_{y} \frac{S_{2}(y)}{(x-y)^{3}} \partial_{y} Z+\right. \\
& \left.+\frac{3}{16} \frac{4 S_{2}(y)+(x-y) S_{2}^{\prime}(y)}{(x-y)^{4}} \partial_{y} Z\right] .
\end{aligned}
$$

Тогда пара (3), (4) коммутирует, если и только если выполнены два следующих условия:

$$
\begin{gathered}
Z_{x, y}=\frac{Z_{x}-Z_{y}}{2(y-x)}, \\
Z_{x} Q_{y}-Z_{y} Q_{x}+R_{1}-R_{2}=0,
\end{gathered}
$$

Докажем это утверждение прямым вычислением. 
Для начала заметим, что производные преобразуются следующим образом:

$$
\left(q_{1}, q_{2}\right) \rightarrow\left(s_{1}, s_{2}\right): \quad \partial_{q_{1}}=-\left(\frac{\Phi_{q_{1}}^{1}}{\Phi_{s_{1}}^{1}} \partial_{s_{1}}+\frac{\Phi_{q_{1}}^{2}}{\Phi_{s_{2}}^{2}} \partial_{s_{2}}\right), \quad \partial_{q_{2}}=-\left(\frac{\Phi_{q_{2}}^{1}}{\Phi_{s_{1}}^{1}} \partial_{s_{1}}+\frac{\Phi_{q_{2}}^{2}}{\Phi_{s_{2}}^{2}} \partial_{s_{2}}\right),
$$

где $\Phi^{i}=\Phi\left(s_{i}, q_{1}, q_{2}\right), i=1,2$. Нетрудно убедиться, что при таком преобразовании перекрестных членов с $\partial_{s_{1}} \partial_{s_{2}}$ в преобразованных операторах $H, K$ нет, а главные (квадратичные) члены имеют такой же вид, как в (3). Функции $S_{i}$ можно вычислить по формуле (в которой переменные $q_{i}$ нужно выразить через $s_{i}$ )

$$
S_{i}\left(s_{i}\right)=\frac{1}{\left(\Phi_{s_{i}}^{i}\right)^{2}}\left[\left(a_{1} s_{i}-a_{2}\right)\left(\Phi_{q_{1}}^{i}\right)^{2}+2\left(b_{1} s_{i}-b_{2}\right) \Phi_{q_{1}}^{i} \Phi_{q_{2}}^{i}+\left(c_{1} s_{i}-c_{2}\right)\left(\Phi_{q_{2}}^{i}\right)^{2}\right] .
$$

Линейные по производным члены можно привести к виду (3) выбором калибровки, остальные члены фиксируются, как и уравнения для функций $S_{1}\left(s_{1}\right), S_{2}\left(s_{2}\right)$, $Z\left(s_{1}, s_{2}\right), f_{1}\left(s_{1}\right), f_{2}\left(s_{2}\right)$. Снова сделаем замену $s_{1}=x, s_{2}=y$, и тем самым утверждение будет доказано.

Конечно, функции $Z(x, y), f_{1}(x), f_{2}(y)$ можно выразить через коэффициенты начальной пары $H, K$, заданной соотношениями (1), однако получающиеся выражения слишком громоздки и мало пригодны для явных вычислений, т. е. целесообразно проводить их отдельно в каждом конкретном случае. Сформулированное утверждение гарантирует, что любую невырожденную пару можно преобразовать к каноническому виду (3), (4). Поэтому в дальнейшем мы будем исследовать именно пары вида (3), (4).

Остановимся еще раз на терминологии: квазиштеккелевой (квантовой) системой мы называем пару (3), (4). Действительно, при переходе к классическому пределу мы получаем классические квазиштеккелевы гамильтонианы, причем при условии $Z(x, y)=0$ эти гамильтонианы переходят в хорошо известные штеккелевы гамильтонианы (заметим, что в этом случае векторные потенциалы, а значит, и магнитное поле равны нулю).

\section{2. ПЕРЕХОД К КВАЗИШТЕККЕЛЕВЫМ ГАМИЛЬТОНИАНАМ}

Вернемся к гамильтонианам (1). Отметим, что в случае уравнения Шредингера

$$
a_{1}\left(q_{1}, q_{2}\right)=1, \quad b_{1}\left(q_{1}, q_{2}\right)=0, \quad c_{1}\left(q_{1}, q_{2}\right)=1 .
$$

Коммутируя гамильтонианы (1) и следя за уравнениями при старших производных, получим, что с точностью до сдвигов и растяжений главные коэффициенты оператора $K$ могут быть выбраны в виде

$$
a_{2}\left(q_{1}, q_{2}\right)=q_{2}^{2}+\mu^{2}, \quad b_{2}\left(q_{1}, q_{2}\right)=-q_{1} q_{2}+\kappa, \quad c_{2}=q_{1}^{2}+\nu^{2} .
$$

Вычисляя $S_{i}$, получим, что в этом случае $S_{1}(t)=S_{2}(t)=S(t)$, где

$$
S(t)=4\left(t-x_{1}\right)\left(t-x_{2}\right), \quad x_{1}=\nu^{2}-\lambda^{2}, \quad x_{2}=\lambda^{2}+\mu^{2}, \quad \kappa^{2}=\lambda^{2}\left(\mu^{2}-\nu^{2}+\lambda^{2}\right) .
$$

Таким образом, в случае уравнения Шредингера функция $S(t)$ есть квадратичный полином, а старшие коэффициенты оператора $K$ и корни этого полинома представляют собой целые функции от трех параметров $\lambda, \mu, \nu$, за исключением коэффициента $b_{2}\left(q_{1}, q_{2}\right)$, содержащего дополнительный параметр $\kappa$, который зависит от параметров $\lambda, \mu, \nu$ через радикал. 
Дальнейшая процедура состоит в следующем. Необходимо при фиксированном полиноме $S(x)$ найти функцию $Z(x, y)$ от двух переменных и функцию $f(x)$ от одной переменной (в нашем случае $f_{1}(x)=f_{2}(x)=f(x)$ ), решая систему $(5),(6)$.

Общее решение уравнения Эйлера-Дарбу (5) можно разложить в ряд по степеням $x-y$ :

$$
Z(x, y)=(x+y) \delta+(x-y)^{2} \sum_{k=0}^{\infty} g^{(2 k)}\left(\frac{x+y}{2}\right) \frac{(x-y)^{2 k}}{2^{2 k} k !(k+1) !} .
$$

Мы опустили несущественную константу, а также ветвь, содержащую $\ln (x-y)$, которая несовместна с уравнением (6) (подробнее см. работу [6]). Подставляя разложение (8) в уравнение (6), разлагая по степеням $x-y$ получившееся уравнение, получаем набор уравнений для $f$ и $g$. Вычисляя вторую производную от $f$ и подставляя ее и старшие производные $f^{(k)}$ в последующие уравнения (они не содержат саму функцию $f$ и ее производную $f^{\prime}$ ), получаем уравнения только относительно $g$, в которые входит также функция $S$.

Оказывается, что первые два нетривиальных уравнения имеют вид

$$
B_{2} \partial_{x}^{4}(g(x) S(x))=0, \quad B_{3} \partial_{x}^{4}(g(x) S(x))=0,
$$

где $B_{2}, B_{3}$ - дифференциальные операторы второго и третьего порядка, которые зависят только от функции $g$ и ее прозводных, но не зависят от $S$ !

Возможны два варианта:

1) имеют место соотношения

$$
\partial_{x}^{4}(g(x) S(x))=0 \Longleftrightarrow g(x)=\frac{P_{3}(x)}{S(x)},
$$

где $P_{3}$ - произвольный полином третьей степени;

2 ) одновременно выполняются уравнения $B_{2} \psi(x)=0$ и $B_{3} \psi(x)=0$.

Во втором случае, исключая последовательно $\psi^{\prime \prime \prime}, \psi^{\prime \prime}, \psi^{\prime}$, получаем уравнение совместности на функцию $g$ вида

$$
g^{V I}=F\left(g^{V}, g^{I V}, g^{\prime \prime \prime}, g^{\prime \prime}, g^{\prime}, g\right) .
$$

Применяя к этому уравнению тест Пенлеве, получаем (с точностью до сдвигов), что оно имеет только изолированные решения

$$
g(t)=-\frac{1}{8} \frac{z}{t}, \quad z=0, \delta, 2 \delta, \pm i \hbar, 2 \delta \pm i \hbar, \delta \pm \frac{1}{2} i \hbar,
$$

или $\delta= \pm 7 \hbar / 2$.

По данной функции $g=-z / 8 t$ легко восстановить и функцию $Z(x, y)$ :

$$
Z(x, y)=(x+y)\left(\delta-\frac{z}{2}\right)+z \sqrt{x} \sqrt{y} .
$$

Конечно, не все полученные решения с данным $z$ являются решениями уравнения (6), но достаточно подставить полученное выражение для $Z$ в (6), найти функцию $f$ и проверить выполнение уравнения (6) точно (без разложения в ряд). 
Рассмотрим подробнее возможные случаи.

Случай 1. Решение с двумя корнями: имеем

$$
\begin{aligned}
Z(x, y)= & \left(\delta-\frac{1}{2}\left(z_{1}+z_{2}\right)\right)(x+y)+\frac{1}{2} z_{\mathrm{m}}(x-y)^{2}(x+y)+z_{\mathrm{c}}(x-y)^{2}+ \\
& +z_{1} \sqrt{x-x_{1}} \sqrt{y-x_{1}}+z_{2} \sqrt{x-x_{2}} \sqrt{y-x_{2}} \\
S(x)= & 4\left(x-x_{1}\right)\left(x-x_{2}\right) \\
f(x)= & -4 z_{\mathrm{m}}^{2} x^{2} S(x)+4 z_{\mathrm{m}} x^{2}\left(z_{1}+z_{2}-2 \delta\right)-16 z_{\mathrm{c}} x^{2}\left(2 z_{\mathrm{m}}\left(x-x_{1}-x_{2}\right)+z_{\mathrm{c}}\right)- \\
& -\frac{z_{1}^{2}}{4} \frac{x_{1}-x_{2}}{x-x_{1}}-\frac{z_{2}^{2}}{4} \frac{x_{2}-x_{1}}{x-x_{2}}
\end{aligned}
$$

где $z_{\mathrm{m}}, z_{\mathrm{c}}$ и далее $f_{\mathrm{m}}, q_{\mathrm{m}}, q_{\mathrm{c}}-$ константы.

Случай 2. Решение с одним корнем: имеем

$$
\begin{aligned}
Z(x, y) & =(x+y)\left(\delta-\frac{z}{2}\right)+z \sqrt{x} \sqrt{y} \\
S(x) & =4\left(x-x_{1}\right)\left(x-x_{2}\right)
\end{aligned}
$$

при $z=0$

$$
f(x)=f_{2} x^{2}
$$

при $z=\delta, x_{1}=0$

$$
f(x)=f_{\mathrm{m}} \sqrt{x}+\frac{\delta^{2}}{4 x} x_{2}
$$

при $z=\delta, \delta= \pm i \hbar$

$$
f(x)=f_{\mathrm{m}} \sqrt{x}+\hbar^{2} \frac{x_{1} x_{2}}{2 x^{2}}-\frac{\hbar^{2}}{4 x}\left(x_{1}+x_{2}\right) ;
$$

при $z=2 \delta$

$$
f(x)=\frac{f_{\mathrm{m}}}{x}-\frac{x_{1} x_{2}}{4 x^{2}}\left(3 \hbar^{2}+8 \delta^{2}\right)
$$

при $z=2 \delta \pm i \hbar$

$$
f(x)=-(2 \delta \pm i \hbar)^{2}\left(\frac{x_{1} x_{2}}{2 x^{2}}-\frac{x_{1}+x_{2}}{4 x}\right) .
$$

Остальные решения, получающиеся из возможных вариантов ответов теста Пенлеве, являются частными случаями вышеперечисленных.

\section{3. ПЕРЕХОД К УРАВНЕНИЮ ШРЕДИНГЕРА. ПРИМЕРЫ.}

Рассмотрим оператор Шредингера в магнитном поле:

$$
H=\widetilde{\pi}_{1}^{2}+\widetilde{\pi}_{2}^{2}+u\left(q_{1}, q_{2}\right)
$$

где

$$
\tilde{\pi}_{1}=-i \hbar \partial_{q_{1}}-\mathcal{A}_{1}\left(q_{1}, q_{2}\right), \quad \tilde{\pi}_{2}=-i \hbar \partial_{q_{2}}-\mathcal{A}_{2}\left(q_{1}, q_{2}\right)
$$


Дополнительный эрмитов интеграл $K$, коммутирующий с $H$, имеет вид

$$
\begin{aligned}
K= & \widetilde{\pi}_{1} a_{2}\left(q_{1}, q_{2}\right) \widetilde{\pi}_{1}+\widetilde{\pi}_{1} b_{2}\left(q_{1}, q_{2}\right) \widetilde{\pi}_{2}+\widetilde{\pi}_{2} b_{2}\left(q_{1}, q_{2}\right) \widetilde{\pi}_{1}+\widetilde{\pi}_{2} c_{2}\left(q_{1}, q_{2}\right) \widetilde{\pi}_{2}+ \\
& +w_{1}\left(q_{1}, q_{2}\right) \widetilde{\pi}_{1}+\widetilde{\pi}_{1} w_{1}\left(q_{1}, q_{2}\right)+w_{2}\left(q_{1}, q_{2}\right) \widetilde{\pi}_{2}+\widetilde{\pi}_{2} w_{2}\left(q_{1}, q_{2}\right)+U\left(q_{1}, q_{2}\right) .
\end{aligned}
$$

Эта пара является канонической формой пары (1), переписанной через операторы скорости. Чтобы перейти от квазиштеккелевой системы (3), (4) к паре (9), (10), необходимо обратить производные и сделать замену переменных. В явном виде это выглядит следующим образом:

$$
\begin{array}{ccc}
\frac{\partial}{\partial x}=-\frac{\Phi_{x}^{1}}{J}\left(\Phi_{q_{2}}^{2} \frac{\partial}{\partial q_{1}}-\Phi_{q_{1}}^{2} \frac{\partial}{\partial q_{2}}\right), & \frac{\partial}{\partial y}=\frac{\Phi_{y}^{2}}{J}\left(\Phi_{q_{2}}^{1} \frac{\partial}{\partial q_{1}}-\Phi_{q_{1}}^{1} \frac{\partial}{\partial q_{2}}\right), \\
\frac{\partial}{\partial s_{1}}=\left.\frac{\partial}{\partial x}\right|_{\substack{x=s_{1} \\
y=s_{2}}}, & \frac{\partial}{\partial s_{2}}=\left.\frac{\partial}{\partial y}\right|_{\substack{x=s_{1}, y=s_{2}}},
\end{array}
$$

где $J=\Phi_{q_{1}}^{1} \Phi_{q_{2}}^{2}-\Phi_{q_{2}}^{1} \Phi_{q_{1}}^{2}$, при этом $\Phi^{1}=\Phi\left(x, q_{1}, q_{2}\right)$ и $\Phi^{2}=\Phi\left(y, q_{1}, q_{2}\right)$, а нижние индексы обозначают производные по соответствующим переменным.

Для упрощения выражений введем новый элемент $Q_{0}$ и запишем $s_{1}, s_{2}$ в виде

$$
s_{1,2}=\frac{1}{2}\left(q_{1}^{2}+q_{2}^{2}+\mu^{2}+\nu^{2} \pm Q_{0}\right),
$$

делая замену $Q_{0} \rightarrow \widetilde{Q}\left(q_{1}, q_{2}\right)$, где

$$
\begin{aligned}
& \widetilde{Q}\left(q_{1}, q_{2}\right)= \\
& =\sqrt{q_{2}^{4}+2 q_{2}^{2} \mu^{2}+2 q_{1}^{2} q_{2}^{2}-2 q_{2}^{2} \nu^{2}+\mu^{4}-2 \mu^{2} q_{1}^{2}+2 \mu^{2} \nu^{2}+q_{1}^{4}+2 q_{1}^{2} \nu^{2}+\nu^{4}-8 q_{1} q_{2} \mu \nu .}
\end{aligned}
$$

Например, замену производных можно записать в виде

$$
\begin{aligned}
\frac{\partial}{\partial s_{1}}= & -\frac{1}{4} \frac{2 q_{1} \kappa+q_{2} \nu^{2}-q_{1}^{2} q_{2}-q_{2}^{3}-q_{2} \mu^{2}+q_{2} Q_{0}}{q_{1} q_{2} \mu^{2}-q_{1} q_{2} \nu^{2}-q_{1}^{2} \kappa+q_{2}^{2} \kappa} \frac{\partial}{\partial q_{1}}+ \\
& +\frac{1}{4} \frac{2 q_{2} \kappa+\mu^{2} q_{1}-q_{1} q_{2}^{2}-q_{1}^{3}-\nu^{2} q_{1}+q_{1} Q_{0}}{q_{1} q_{2} \mu^{2}-q_{1} q_{2} \nu^{2}-q_{1}^{2} \kappa+q_{2}^{2} \kappa} \frac{\partial}{\partial q_{2}}+\frac{\partial}{\partial Q_{0}} \\
\frac{\partial}{\partial s_{2}}= & \frac{1}{4} \frac{q_{1}^{2} q_{2}-2 q_{1} \kappa-q_{2} \nu^{2}+q_{2}^{3}+q_{2} \mu^{2}+q_{2} Q_{0}}{q_{1} q_{2} \mu^{2}-q_{1} q_{2} \nu^{2}-q_{1}^{2} \kappa+q_{2}^{2} \kappa} \frac{\partial}{\partial q_{1}}- \\
& -\frac{1}{4} \frac{q_{1} q_{2}^{2}-2 q_{2} \kappa-\mu^{2} q_{1}+q_{1}^{3}+\nu^{2} q_{1}+q_{1} Q_{0}}{q_{1} q_{2} \mu^{2}-q_{1} q_{2} \nu^{2}-q_{1}^{2} \kappa+q_{2}^{2} \kappa} \frac{\partial}{\partial q_{2}}-\frac{\partial}{\partial Q_{0}} .
\end{aligned}
$$

Дальнейшая процедура является чисто технической и заключается в замене производных, окончательной замене переменных $\left(s_{1}, s_{2}\right) \rightarrow\left(q_{1}, q_{2}\right)$ в паре $(3),(4)$ и приведении получившихся выражений к каноническому виду (9), (10).

Следует отметить, что описанная процедура позволяет получить все двумерные уравнения Шредингера (невырожденые в смысле раздела 1), однако получающиеся выражения чрезвычайно громоздки. В случае полной $(\lambda, \mu, \nu)$-параметризации вычислительной мощности не хватает для всех пар, поэтому в настоящей статье мы приведем лишь несколько примеров. 
Пример 1. В случае полной $(\lambda, \mu, \nu)$-параметризации наиболее компактным является случай с одним корнем, который уже рассматривался в работе [5]. Мы рассмотрим его здесь в другой параметризации и с вещественной структурой:

$$
\begin{aligned}
Z(x, y) & =2 i \delta \sqrt{x} \sqrt{y} \\
S(t) & =4\left(t-x_{1}\right)\left(t-x_{2}\right), \\
f(t) & =-\alpha \frac{3 \hbar^{2}-8 \delta^{2}}{4 t^{2}}+\frac{f_{\mathrm{m}}-2 \delta^{2}\left(\mu^{2}+\nu^{2}\right)}{t} .
\end{aligned}
$$

Тогда

$$
\begin{gathered}
\mathcal{A}_{1}(x, y)=-\delta \frac{R_{y} G-G_{y} R}{G^{2}-4 R^{2}}, \quad \mathcal{A}_{2}(x, y)=\delta \frac{R_{x} G-G_{x} R}{G^{2}-4 R^{2}}, \quad B(x, y)=\delta \frac{\alpha}{R^{3}}, \\
u(x, y)=-\frac{f_{\mathrm{m}}}{2 R^{2}}+\frac{3}{4} \hbar^{2} \alpha \frac{G}{R^{4}}, \quad w_{1}(x, y)=\delta R_{y}, \quad w_{2}(x, y)=-\delta R_{x}, \\
U(x, y)=-f_{\mathrm{m}} \frac{G}{2 R^{2}}+\frac{3}{4} \hbar^{2} \frac{\alpha}{R^{2}}+\frac{3}{4} \hbar^{2} \alpha \frac{G^{2}}{R^{4}},
\end{gathered}
$$

где

$\alpha=x_{1} x_{2}=\mu^{2} \nu^{2}-\kappa^{2}, \quad R=\sqrt{\mu^{2} x^{2}+\nu^{2} y^{2}+2 \kappa x y+\alpha}, \quad G=x^{2}+y^{2}+\mu^{2}+\nu^{2}$.

ПримеР 2. Для $(\mu, \nu)$-параметризации вычисления дают, что системы, рассмотренные в случае 2 предыдущего раздела, приводят к тривиальным системам с уже разделенными переменными и легко интегрируются. Поэтому рассмотрим случай 1 с максимально возможным числом параметров: наложим единственное ограничение $z_{2}=0$. Имеем

$$
\begin{aligned}
Z(x, y)= & -i \delta\left(\frac{1}{2}(x+y)+\sqrt{x} \sqrt{y}\right)+(x-y)^{2}\left(\frac{1}{2} q_{\mathrm{m}}(x+y)+q_{\mathrm{c}}\right), \\
S(x)= & 4 x\left(x-\left(\mu^{2}+\nu^{2}\right)\right), \\
f(x)= & 16 q_{\mathrm{m}}^{2} x^{3}\left(x-\mu^{2}-\nu^{2}\right)+4 q_{\mathrm{m}} x^{2} \delta+16 q_{\mathrm{c}} x^{2}\left(2 q_{\mathrm{m}}\left(x-\mu^{2}-\nu^{2}\right)+q_{\mathrm{c}}\right)- \\
& -\frac{1}{4}\left(\mu^{2}+\nu^{2}\right) \frac{\delta^{2}}{x} .
\end{aligned}
$$

Тогда

$$
\begin{gathered}
\mathcal{A}_{1}(x, y)=-\frac{1}{2} q_{\mathrm{m}}\left(\nu^{2} y-2 x \nu \mu+3 y \mu^{2}+3 x^{2} y+3 y^{3}\right)-2 q_{\mathrm{c}} y, \\
\mathcal{A}_{2}(x, y)=\frac{1}{2} q_{\mathrm{m}}\left(3 \nu^{2} x+3 x^{3}+\mu^{2} x-2 \mu \nu y+3 y^{2} x\right)+2 q_{\mathrm{c}} x, \\
B(x, y)=2 q_{\mathrm{m}}\left(3\left(x^{2}+y^{2}\right) \mu^{2}+\nu^{2}\right)+4 q_{\mathrm{c}}, \\
u(x, y)=2\left(-q_{\mathrm{m}}^{2}\left(y^{2}+\mu^{2}+x^{2}+\nu^{2}\right)-2 q_{\mathrm{m}} q_{\mathrm{c}}\right) \times \\
\times\left(\nu^{2}+\mu^{2}-2 \mu x+x^{2}-2 y \nu+y^{2}\right)\left(\nu^{2}+\mu^{2}+2 \mu x+x^{2}+2 y \nu+y^{2}\right)- \\
-q_{\mathrm{m}} \delta\left(-2 \mu^{2}-2 \nu^{2}+2 x^{2}+2 y^{2}+4 y \nu+4 \mu x\right),
\end{gathered}
$$




$$
\begin{aligned}
v_{1}(x, y)= & -\frac{1}{2} q_{\mathrm{m}}\left(3 \mu^{4} y-4 \mu^{3} \nu x+2 \mu^{2} \nu^{2} y+2 \mu^{2} x^{2} y+6 \mu^{2} y^{3}-4 \mu \nu^{3} x-4 \mu \nu x^{3}-\right. \\
& \left.-12 \mu \nu x y^{2}-\nu^{4} y+2 \nu^{2} x^{2} y-2 \nu^{2} y^{3}+3 x^{4} y+6 x^{2} y^{3}+3 y^{5}\right)- \\
& -2 q_{\mathrm{c}}\left(y \mu^{2}-2 x \nu \mu-\nu^{2} y+x^{2} y+y^{3}\right)-\frac{1}{2} \delta(y+\nu), \\
v_{2}(x, y)= & -\frac{1}{2} q_{\mathrm{m}}\left(\mu^{4} x+4 \mu^{3} \nu y-2 \mu^{2} \nu^{2} x+2 \mu^{2} x^{3}-2 \mu^{2} x y^{2}+4 \mu \nu^{3} y+4 \mu \nu y^{3}+\right. \\
& \left.+12 \mu \nu x^{2} y-3 \nu^{4} x-6 \nu^{2} x^{3}-2 \nu^{2} x y^{2}-3 x^{5}-6 x^{3} y^{2}-3 x y^{4}\right)- \\
& -2 q_{\mathrm{c}}\left(\mu^{2} x+2 \mu \nu y-\nu^{2} x-x^{3}-y^{2} x\right)+\frac{1}{2} \delta(x+\mu), \\
U(x, y)= & \left(\frac { 1 } { 4 } q _ { \mathrm { m } } ^ { 2 } \left(\nu^{4}+10 x^{2} \nu^{2}+6 y^{2} \nu^{2}+2 \mu^{2} \nu^{2}-8 \nu x y \mu+9 x^{4}+9 y^{4}+\mu^{4}+\right.\right. \\
& \left.\left.+18 x^{2} y^{2}+10 y^{2} \mu^{2}+6 \mu^{2} x^{2}\right)+4 q_{\mathrm{c}}^{2}+2 q_{\mathrm{c}} q_{\mathrm{m}}\left(\nu^{2}+\mu^{2}+3\left(x^{2}+y^{2}\right)\right)\right) \times \\
& \times\left(\nu^{2}+\mu^{2}-2 \mu x+x^{2}-2 y \nu+y^{2}\right)\left(\nu^{2}+\mu^{2}+2 \mu x+x^{2}+2 y \nu+y^{2}\right)- \\
& -\frac{1}{2} q_{\mathrm{m}} \delta\left(\nu^{2}+\mu^{2}+2 \mu x-3 x^{2}+2 y \nu-3 y^{2}\right)\left(\nu^{2}+\mu^{2}+2 \mu x+x^{2}+2 y \nu+y^{2}\right)- \\
& -q_{\mathrm{c}} \delta\left(-2 y^{2}-2 x^{2}-4 y \nu-4 \mu x+2 \mu^{2}+2 \nu^{2}\right) .
\end{aligned}
$$

Попытка найти все пары вида (1), непосредственно коммутируя их, приводит к тому, что при фиксированных коэффициентах $a_{1}=1, b_{1}=0, c_{1}=1$ (в случае уравнения Шредингера) можно определить старшие коэффициенты дополнительного интеграла $K(7)$. Остальные коэффициенты гамильтонианов $H, K$ определяются дифференциальными уравнениями с частными производными, которые не решаются в общем виде. Поэтому необходимо найти все коммутирующие пары квазиштеккелевых систем (3), (4) и провести замену переменных $\left(s_{1}, s_{2}\right) \rightarrow\left(q_{1}, q_{2}\right)$. Таким образом можно получить полную классификацию уравнений Шредингера в электромагнитном поле с дополнительным (квадратичным) интегралом движения.

Остаются нерешенными вопросы о разделении переменных в рассматриваемых системах и решении получающихся уравнений Шредингера. Возможно, для этого потребуется решать квазиштеккелевы квантовые уравнения $H \psi=\lambda \psi, K \psi=\mu \psi$, a затем проводить замену переменных. Следует отметить, что штеккелевы квантовые системы позволяют простым образом найти разделение переменных и проинтегрировать уравнения. В общем (квазиштекелевом) виде может помочь квазиклассическое разложение. Например, квазиклассическая волновая функция имеет вид

$$
\psi \sim e^{J / i \hbar} \frac{(x-y)^{3 / 2}}{\sqrt{\left[(x-y)^{2}\left(\partial_{x} J\right)\left(\partial_{y} J\right)+\left(\partial_{x} Z\right)\left(\partial_{y} Z\right)\right] S(x) S(y)}},
$$

где $J=J(x, y)$ - функция Гамильтона-Якоби квазиштекеллевой системы.

Благодарности. Автор признателен В. Е. Адлеру, В. В. Соколову и А.Б. Шабату за полезные обсуждения. Работа выполнена при поддержке РФФИ (грант № 13-01-00088). 


\section{Список литературы}

[1] E. V. Ferapontov, A. P. Veselov, J. Math. Phys., 42:2 (2001), 590-607.

[2] Б. А. Дубровин, И. М. Кричевер, С. П. Новиков, Докл. АН СССР, 229 (1976), 15-18.

[3] S. P. Novikov, A. P. Veselov, Amer. Math. Soc. Transl. Ser. 2, 179 (1997), 109-132.

[4] J. Berube, P. Winternitz, J. Math. Phys., 45:5 (2004), 1959-1973.

[5] В. Г. Марихин, Писъма в ЖЭТФ, 94:3 (2011), 262-266.

[6] В. Г. Марихин, В. В. Соколов, ТМФ, 149:2 (2006), 147-160.

[7] В. Г. Марихин, Писъма в ЖЭЭФ, 97:7 (2013), 491-495.

[8] L. P. Eisenhart, Ann. Math., 35:2 (1934), 284-305. 